\begin{tabular}{|c|l|}
\hline Title & Gate voltage induced switching of the Rashba spin-orbit interaction in a composition-adjusted quantum well \\
\hline Author(s) & A kera, Hiroshi; Suzuura, Hidekatsu; Egami, Y oshiyuki \\
\hline Citation & $\begin{array}{l}\text { Physical Review B, 95(4), 045301 } \\
\text { https://doi.org/10.1103/PhysRevB.95.045301 }\end{array}$ \\
\hline Issue Date & 2017-01-03 \\
\hline Doc URL & http://hdl.handle.net/2115/64559 \\
\hline Rights & @2017 A merican Physical Society \\
\hline Type & article \\
\hline File Information & PhysRevB.95.045301.pdf \\
\hline
\end{tabular}

Instructions for use 


\title{
Gate-voltage-induced switching of the Rashba spin-orbit interaction in a composition-adjusted quantum well
}

\author{
Hiroshi Akera, Hidekatsu Suzuura, and Yoshiyuki Egami \\ Division of Applied Physics, Faculty of Engineering, Hokkaido University, Sapporo, Hokkaido 060-8628, Japan
}

(Received 11 May 2016; revised manuscript received 10 November 2016; published 3 January 2017)

\begin{abstract}
The coefficient $\alpha$ of the Rashba spin-orbit interaction is calculated in an asymmetric quantum well consisting of $\mathrm{Ga}_{0.47} \mathrm{In}_{0.53}$ As (well), $\mathrm{Al}_{0.48} \mathrm{In}_{0.52} \mathrm{As}$ (left barrier), and $\mathrm{Al}_{x} \mathrm{Ga}_{1-x} \mathrm{As}_{y} \mathrm{Sb}_{1-y}$ (right barrier) as a function of the external electric field perpendicular to the well $E_{z}^{\text {ex }}$ which is controlled by the gate voltage. This coefficient $\alpha$, which depends on the band offset, can be tuned to be zero by adjusting the Al fraction $x$ in the right barrier layer to the optimum value $x_{0}$ in the case where the wave function vanishes at the left heterointerface. Such a composition-adjusted asymmetric quantum well is proposed as a structure in which the magnitude of $\alpha$ can be switched by changing the polarity of $E_{z}^{\mathrm{ex}}$. The calculation shows that, when $\left|x-x_{0}\right|<0.01$, the on/off $|\alpha|$ ratio $>40$ for a large enough $\left|E_{z}^{\mathrm{ex}}\right|\left(\left|E_{z}^{\mathrm{ex}}\right|>10^{7} \mathrm{~V} / \mathrm{m}\right.$ for a well width of $\left.20 \mathrm{~nm}\right)$, which results in the on/off spin-relaxation-rate ratio exceeding $10^{3}$ in the Dyakonov-Perel mechanism.
\end{abstract}

DOI: 10.1103/PhysRevB.95.045301

Spintronics [1,2], which explores the application of the spin degree of freedom to electronics, is one of the major subjects in applied physics. As a milestone in spintronics, Datta and Das have proposed a spin field-effect transistor (FET) [3], in which the spin orientation of an electron, in transport through a two-dimensional channel, rotates around the in-plane effective magnetic field $\left(\boldsymbol{B}_{\text {eff }}\right)$ induced by the Rashba spin-orbit interaction [4-7]. The angular frequency of the spin rotation is proportional to $B_{\text {eff }}\left(\equiv\left|\boldsymbol{B}_{\text {eff }}\right|\right)$. This effective magnetic field switches the current on and off each time the change of $B_{\text {eff }}$ increases the angle of the spin rotation in the channel by $\pi$. Therefore the action of this spin FET relies on the control of $B_{\text {eff }}$ by the gate voltage, that is, by the external electric field perpendicular to the plane $E_{z}^{\mathrm{ex}}$, which has been confirmed by experiments in quantum wells [8-10].

Another spin FET [11,12], called the spin-lifetime FET, has been proposed by Hall and others in which the spin relaxation switches the current on by changing the electron distribution from fully spin-polarized to unpolarized. The spin-lifetime FET, in contrast to the Datta-Das spin FET, uses only one component of the spin polarization vector. The action of the spin-lifetime FET relies on the switching of the spin relaxation rate by the change of the gate voltage. As a method to vary the spin-relaxation rate, the original paper $[11,12]$ proposed to use the above-mentioned $E_{z}^{\text {ex }}$ dependence of $B_{\text {eff }}\left(\propto\left|E_{z}^{\text {ex }}\right|\right)$ in quantum wells [8-10], which makes the spin-relaxation rate in the Dyakonov-Perel mechanism [13-15] $\left[\propto\left(B_{\text {eff }}\right)^{2}\right]$ proportional to $\left(E_{z}^{\text {ex }}\right)^{2}$. The decrease of $E_{z}^{\mathrm{ex}}$ from a higher value $E_{z}^{\mathrm{H}}$ to a lower one $E_{z}^{\mathrm{L}}$ reduces the current from $I_{\mathrm{on}} \propto\left(E_{z}^{\mathrm{H}}\right)^{2}$ to $I_{\mathrm{off}} \propto\left(E_{z}^{\mathrm{L}}\right)^{2}$, leading to the on/off current ratio $I_{\mathrm{on}} / I_{\mathrm{off}}=\left(E_{z}^{\mathrm{H}} / E_{z}^{\mathrm{L}}\right)^{2}$. Unfortunately, this method requires a precise control of the gate voltage to achieve a high on/off current ratio because $E_{z}^{\mathrm{L}}$ must be in the close vicinity of $E_{z}^{\mathrm{ex}}=0$.

We have recently found [16] that $B_{\text {eff }}$ due to the Rashba spin-orbit interaction and the resulting spin-relaxation rate vary in a wide range by changing the band offsets (of conduction, valence, and split-off bands) between the well and barrier semiconductors in a quantum well. In particular, the coefficient $\alpha$ of the Rashba spin-orbit interaction (and of
$\left.\boldsymbol{B}_{\text {eff }}\right)$ can be tuned to vanish by adjusting the band offsets. For example, in a quantum well consisting of $\mathrm{Ga}_{0.47} \mathrm{In}_{0.53} \mathrm{As}$ (well) and $\mathrm{Al}_{x} \mathrm{Ga}_{1-x} \mathrm{As}_{y} \mathrm{Sb}_{1-y}$ (barrier), where the band offsets change with the $\mathrm{Al}$ fraction $x, \alpha$ and the associated spin-relaxation rate become zero at an optimum fraction $x_{0}$.

In this paper we propose and explore the gate-voltageinduced switching of the Rashba coefficient $\alpha$ by using the above-mentioned band-offset dependence of $\alpha$. The first key factor of the proposed switching is an asymmetric quantum-well structure with two barrier layers formed by different semiconductors, $\mathrm{Al}_{x} \mathrm{Ga}_{1-x} \mathrm{As}_{y} \mathrm{Sb}_{1-y}\left(x \approx x_{0}\right)$ and $\mathrm{Al}_{0.48} \mathrm{In}_{0.52}$ As. This combination of barrier semiconductors is chosen so that one (the other) interface is that in a symmetric quantum well with a small $|\alpha|$ (a large $|\alpha|$ ). The second is the wave function deformation due to the external electric field $E_{z}^{\mathrm{ex}}$, which is produced by the gate voltage. Then the gate voltage switches $|\alpha|$ on and off as the external electric field moves the wave function to the interface with a large $|\alpha|$ and to that with a small $|\alpha|$. We examine the on/off $|\alpha|$ ratio when the $\mathrm{Al}$ fraction $x$ in $\mathrm{Al}_{x} \mathrm{Ga}_{1-x} \mathrm{As}_{y} \mathrm{Sb}_{1-y}$ deviates from the optimum value $x_{0}$ and investigate the required $\left|E_{z}^{\mathrm{ex}}\right|$.

We consider an electron in the conduction band of a quantum-well structure which is formed by three different semiconductors with the zinc-blende structure (Fig. 1): $S_{B}^{L}$ in the left barrier layer $\left(z<z_{\mathrm{I}}^{\mathrm{L}}\right), \mathrm{S}_{\mathrm{W}}$ in the well layer $\left(z_{\mathrm{I}}^{\mathrm{L}}<z<\right.$ $\left.z_{\mathrm{I}}^{\mathrm{R}}\right)$, and $\mathrm{S}_{\mathrm{B}}^{\mathrm{R}}$ in the right barrier layer $\left(z_{\mathrm{I}}^{\mathrm{R}}<z\right)$. Due to the translational symmetry along the $x$ and $y$ axes, the associated wave numbers, $k_{x}$ and $k_{y}$, are conserved. The Schrödinger equation for the ground-subband wave function $\varphi_{0}(z)$ and the corresponding eigenvalue $\varepsilon_{0}$ is

$$
\left[\frac{\hat{p}_{z}^{2}}{2 m}+V_{\mathrm{W}}(z)\right] \varphi_{0}(z)=\varepsilon_{0} \varphi_{0}(z),
$$

where $\hat{p}_{z}=-i \hbar \nabla_{z}=-i \hbar \partial / \partial z$ and $m$ is the effective mass of the conduction band. The confining potential $V_{\mathrm{W}}(z)$ is

$$
V_{\mathrm{W}}(z)=V_{\mathrm{bo}}^{\mathrm{c}}(z)+V_{\mathrm{es}}(z) .
$$

Here $V_{\mathrm{bo}}^{\mathrm{c}}(z)$ is the potential due to the conduction-band offset (Fig. 1), the expression of which is given later with those for valence bands. The second term $V_{\mathrm{es}}(z)$, which is the 


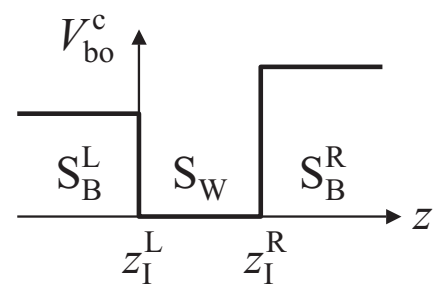

FIG. 1. A quantum-well structure consisting of three different semiconductors with the zinc-blende structure, $\mathrm{S}_{\mathrm{B}}^{\mathrm{L}}, \mathrm{S}_{\mathrm{W}}$, and $\mathrm{S}_{\mathrm{B}}^{\mathrm{R}} \cdot V_{\mathrm{bo}}^{\mathrm{c}}(z)$ is the potential due to the conduction-band offset.

electrostatic potential due to the charge distribution in the quantum-well structure, satisfies the Poisson equation

$$
\nabla_{z}^{2} V_{\mathrm{es}}=-A n(z)
$$

where

$$
A=\frac{4 \pi e^{2}}{\epsilon}, \quad n(z)=N_{\mathrm{s}}\left[\varphi_{0}(z)\right]^{2},
$$

with $\epsilon$ the static dielectric constant, $-e$ the electron charge $(e>0)$, and $N_{\mathrm{S}}$ the sheet density of electrons. The total electric field is $E_{z}=\nabla_{z} V_{\text {es }} / e$, while the external electric field $E_{z}^{\text {ex }}$ is that induced by ionized donors and charge induced on the gate electrode. Such charges, which are placed in $z<z_{\mathrm{d}}^{\mathrm{L}}$ and $z>z_{\mathrm{d}}^{\mathrm{R}}$, are assumed to be far away from the quantum well so that $E_{z}^{\text {ex }}$ acting on electrons is constant (this assumption can be satisfied by employing the widely used modulation doping where the spacer layer thicker than $10 \mathrm{~nm}$ is inserted between the well layer and each doped layer so that the penetration of the wave function into each doped layer is negligible). We introduce the areal external-charge density in the left side of the well $\left(z<z_{\mathrm{d}}^{\mathrm{L}}\right), \sigma_{\mathrm{L}}$, and that in the right side $\left(z>z_{\mathrm{d}}^{\mathrm{R}}\right), \sigma_{\mathrm{R}}$. Then the boundary condition for $V_{\mathrm{es}}$ at $z=z_{\mathrm{d}}^{\mathrm{L}}$ becomes

$$
\nabla_{z} V_{\mathrm{es}}=A\left(\sigma_{\mathrm{L}} / e\right) \quad\left(z=z_{\mathrm{d}}^{\mathrm{L}}\right) .
$$

The charge neutrality gives $e N_{\mathrm{s}}=\sigma_{\mathrm{L}}+\sigma_{\mathrm{R}}$, while $E_{z}^{\mathrm{ex}}=$ $(2 \pi / \epsilon)\left(\sigma_{\mathrm{L}}-\sigma_{\mathrm{R}}\right)$.

We derive the formula for the spin-orbit interaction induced by the band offsets and the electrostatic potential, which is denoted by $V_{\mathrm{W}}^{\text {so }}$, as well as the Rashba coefficient $\alpha$ in the case of an asymmetric quantum well consisting of three different semiconductors [17]. In deriving $V_{\mathrm{W}}^{\text {so }}$ we employ the $\boldsymbol{k} \cdot \boldsymbol{p}$ theory developed for heterostructures [18,19] (it has been shown in a number of papers $[9,10,20-24]$ that the Rashba coefficient $\alpha$ derived by the $\boldsymbol{k} \cdot \boldsymbol{p}$ theory in various heterostructures agrees well with the experimental value and with that calculated in the tight-binding model). Then we obtain, for an electron with wave numbers $k_{x}$ and $k_{y}$ in the conduction band,

$$
V_{\mathrm{W}}^{\mathrm{so}}=\frac{P^{2}}{3} G(z)\left(\sigma_{x} k_{y}-\sigma_{y} k_{x}\right),
$$

where $\sigma_{x}$ and $\sigma_{y}$ are the Pauli spin matrices and $P$ is the Kane matrix element [25]. In this equation,

$$
G(z)=\nabla_{z}\left(\frac{1}{E-\tilde{E}_{\mathrm{v}}}-\frac{1}{E-\tilde{E}_{\mathrm{s}}}\right),
$$

where $E$ is the electron energy measured from the conductionband bottom of $\mathrm{S}_{\mathrm{W}}$, while $\widetilde{E}_{\mathrm{v}}\left(\tilde{E}_{\mathrm{S}}\right)$ is the diagonal element of the $8 \times 8$ Kane Hamiltonian, corresponding to the heavy-hole plus light-hole bands (the split-off band). They are given by

$$
\begin{aligned}
& \tilde{E}_{\mathrm{v}}=E_{\mathrm{K} 0}-E_{\mathrm{g}}+V_{\mathrm{bo}}^{\mathrm{v}}(z)+V_{\mathrm{es}}(z), \\
& \tilde{E}_{\mathrm{s}}=E_{\mathrm{K} 0}-E_{\mathrm{g}}^{\mathrm{s}}+V_{\mathrm{bo}}^{\mathrm{s}}(z)+V_{\mathrm{es}}(z) .
\end{aligned}
$$

Here $E_{\mathrm{K} 0}=\left(\hbar^{2} / 2 m_{0}\right)\left(k_{x}^{2}+k_{y}^{2}+\hat{k}_{z}^{2}\right)$ with $m_{0}$ the electron rest mass and $\hat{k}_{z}=-i \nabla_{z}$, and $E_{\mathrm{g}}^{\mathrm{s}}=E_{\mathrm{g}}+\Delta_{\text {so }}$ with $E_{\mathrm{g}}\left(\Delta_{\mathrm{so}}\right)$ the band gap (the spin-orbit splitting) of $S_{\mathrm{W}}$, while $V_{\mathrm{bo}}^{\mathrm{v}}(z)\left[V_{\mathrm{bo}}^{\mathrm{s}}(z)\right]$ is the potential due to the band offset for an electron in the heavy-hole plus light-hole bands [the split-off band]. These potentials together with that for the conduction band, $V_{\mathrm{bo}}^{\mathrm{c}}(z)$ in Eq. (2), are expressed by

$$
V_{\mathrm{bo}}^{i}(z)=V_{\mathrm{bo}}^{i \mathrm{~L}}(z)+V_{\mathrm{bo}}^{i \mathrm{R}}(z), \quad V_{\mathrm{bo}}^{i \ell}(z)=\Delta E_{i}^{\ell} h_{\ell}(z),
$$

where $i=\mathrm{c}, \mathrm{v}, \mathrm{s}, \ell=\mathrm{L}, \mathrm{R}$, and

$$
h_{\mathrm{L}}(z)=\left\{\begin{array}{ll}
1 & \left(z<z_{\mathrm{I}}^{\mathrm{L}}\right), \\
0 & \left(z>z_{\mathrm{I}}^{\mathrm{L}}\right),
\end{array} \quad h_{\mathrm{R}}(z)= \begin{cases}0 & \left(z<z_{\mathrm{I}}^{\mathrm{R}}\right), \\
1 & \left(z>z_{\mathrm{I}}^{\mathrm{R}}\right),\end{cases}\right.
$$

while $\Delta E_{\mathrm{c}}^{\ell}, \Delta E_{\mathrm{v}}^{\ell}$, and $\Delta E_{\mathrm{s}}^{\ell}$ are the band offsets of the semiconductor $\mathrm{S}_{\mathrm{B}}^{\ell}$ relative to $\mathrm{S}_{\mathrm{W}}: \Delta E_{\mathrm{c}}^{\ell}=E_{\mathrm{c}}^{\ell}-E_{\mathrm{c}}, \Delta E_{\mathrm{v}}^{\ell}=$ $E_{\mathrm{v}}^{\ell}-E_{\mathrm{v}}$, and $\Delta E_{\mathrm{s}}^{\ell}=E_{\mathrm{s}}^{\ell}-E_{\mathrm{s}}$ with $E_{\mathrm{c}}\left(E_{\mathrm{c}}^{\ell}\right)$ the energy of the conduction-band bottom, $E_{\mathrm{v}}\left(E_{\mathrm{v}}^{\ell}\right)$ that of the valance-band top, $E_{\mathrm{s}}\left(E_{\mathrm{s}}^{\ell}\right)$ that of the split-off-band top in $\mathrm{S}_{\mathrm{W}}\left(\right.$ in $\left.\mathrm{S}_{\mathrm{B}}^{\ell}\right)$. We can also express $E_{\mathrm{g}}$ and $\Delta_{\mathrm{so}}$ as $E_{\mathrm{g}}=E_{\mathrm{c}}-E_{\mathrm{v}}$ and $\Delta_{\mathrm{so}}=E_{\mathrm{v}}-E_{\mathrm{s}}$.

Here we neglect $E, \quad E_{\mathrm{K} 0}$, and $V_{\mathrm{es}}(z)$ in $G(z)$, compared to $E_{\mathrm{g}}$ and $E_{\mathrm{g}}^{\mathrm{s}}$, while we take into account the contribution from $\nabla_{z} V_{\mathrm{es}}(z)$ to $G(z)$, that is, $\left[\left(E_{\mathrm{g}}-V_{\mathrm{bo}}^{\mathrm{v}}\right)^{-2}-\left(E_{\mathrm{g}}^{\mathrm{s}}-V_{\mathrm{bo}}^{\mathrm{s}}\right)^{-2}\right] \nabla_{z} V_{\mathrm{es}}$, in which we neglect $V_{\mathrm{bo}}^{\mathrm{v}}$ and $V_{\mathrm{bo}}^{\mathrm{s}}$ since they are nonzero only in the barrier layers where the squared wave function is small (we have confirmed by the numerical calculation that the correction due to $V_{\mathrm{bo}}^{\mathrm{v}}$ and $V_{\text {bo }}^{\mathrm{s}}$ in this contribution is less than 6 percent in the cases we considered in this paper). We finally obtain the expression for $V_{\mathrm{W}}^{\mathrm{so}}$ :

$$
V_{\mathrm{W}}^{\mathrm{so}}=a(z)\left(\sigma_{x} k_{y}-\sigma_{y} k_{x}\right),
$$

with

$$
a(z)=\eta\left[\nabla_{z}\left(b_{\mathrm{off}}^{\mathrm{L}} V_{\mathrm{bo}}^{\mathrm{cL}}+b_{\mathrm{off}}^{\mathrm{R}} V_{\mathrm{bo}}^{\mathrm{cR}}+V_{\mathrm{es}}\right)\right] .
$$

Here $\eta$ is the effective coupling constant of the spin-orbit interaction for an electron in the conduction band of the semiconductor $S_{\mathrm{W}}$, given by

$$
\eta=\frac{P^{2}}{3}\left[\frac{1}{\left(E_{\mathrm{g}}\right)^{2}}-\frac{1}{\left(E_{\mathrm{g}}^{\mathrm{s}}\right)^{2}}\right],
$$

and $b_{\mathrm{off}}^{\ell}(\ell=\mathrm{L}, \mathrm{R})$ is defined by [16]

$$
b_{\mathrm{off}}^{\ell}=\frac{\Delta E_{\mathrm{v}}^{\ell} /\left[E_{\mathrm{g}}\left(E_{\mathrm{g}}-\Delta E_{\mathrm{v}}^{\ell}\right)\right]-\Delta E_{\mathrm{s}}^{\ell} /\left[E_{\mathrm{g}}^{\mathrm{s}}\left(E_{\mathrm{g}}^{\mathrm{s}}-\Delta E_{\mathrm{s}}^{\ell}\right)\right]}{\Delta E_{\mathrm{c}}^{\ell}\left\{1 /\left(E_{\mathrm{g}}\right)^{2}-1 /\left(E_{\mathrm{g}}^{\mathrm{s}}\right)^{2}\right\}} \text {. }
$$

Equation (11) with (12) shows that the spin-orbit interaction $V_{\mathrm{W}}^{\text {so }}$ for an electron in the conduction band, due to the band offsets and the electrostatic potential, is not proportional to $\nabla_{z} V_{\mathrm{W}}$ except the case where $b_{\text {off }}^{\mathrm{L}}=1$ and $b_{\text {off }}^{\mathrm{R}}=1$.

The Rashba coefficient $\alpha$ is defined by the expectation value of $a(z)$ with respect to $\varphi_{0}(z)=\langle z \mid 0\rangle$,

$$
\alpha=\langle 0|a(z)| 0\rangle .
$$




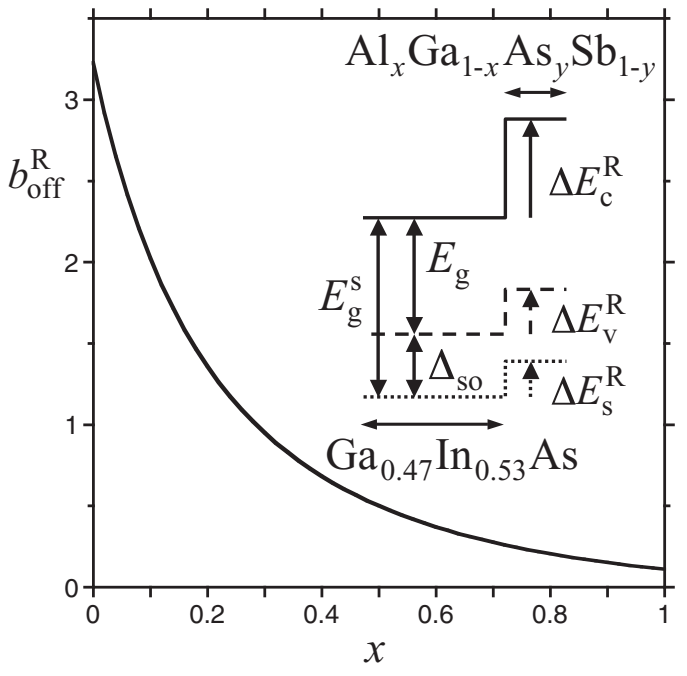

FIG. 2. The factor $b_{\text {off }}^{\mathrm{R}}$ [Eq. (14)] for $\mathrm{S}_{\mathrm{W}}=\mathrm{Ga}_{0.47} \mathrm{In}_{0.53}$ As and $\mathrm{S}_{\mathrm{B}}^{\mathrm{R}}=\mathrm{Al}_{x} \mathrm{Ga}_{1-x} \mathrm{As}_{y} \mathrm{Sb}_{1-y}$ as a function of the $\mathrm{Al}$ fraction $x$ in $\mathrm{S}_{\mathrm{B}}^{\mathrm{R}}$ [16]. We have used band parameters in Ref. [30] and employed the linear interpolation of band offsets between $x=0$ and $x=1$ to obtain $\Delta E_{\mathrm{c}}^{\mathrm{R}}[\mathrm{eV}]=0.436+1.43 x, \Delta E_{\mathrm{v}}^{\mathrm{R}}[\mathrm{eV}]=0.444-0.354 x$, and $\Delta E_{\mathrm{s}}^{\mathrm{R}}[\mathrm{eV}]=0.373-0.371 x$, corresponding to $\mathrm{Ga}_{0.47} \mathrm{In}_{0.53} \mathrm{As}$, $E_{\mathrm{g}}[\mathrm{eV}]=0.816$, and $\Delta_{\mathrm{so}}[\mathrm{eV}]=0.330$.

Here we use the equality $\left\langle 0\left|\left(\nabla_{z} V_{\mathrm{W}}\right)\right| 0\right\rangle=0$, which means that forces on an electron in a bound eigenstate are balanced [26,27] (the equality is derived according to [28] in [29]). Then we obtain the expression of $\alpha$ using values of the wave function at interfaces:

$$
\begin{aligned}
\alpha= & \eta\left(b_{\mathrm{off}}^{\mathrm{L}}-1\right)\left\langle 0\left|\left(\nabla_{z} V_{\mathrm{bo}}^{\mathrm{cL}}\right)\right| 0\right\rangle+\eta\left(b_{\mathrm{off}}^{\mathrm{R}}-1\right)\left\langle 0\left|\left(\nabla_{z} V_{\mathrm{bo}}^{\mathrm{cR}}\right)\right| 0\right\rangle \\
= & \eta\left(b_{\mathrm{off}}^{\mathrm{L}}-1\right)\left[\varphi_{0}\left(z_{\mathrm{I}}^{\mathrm{L}}\right)\right]^{2}\left(-\Delta E_{\mathrm{c}}^{\mathrm{L}}\right)+\eta\left(b_{\mathrm{off}}^{\mathrm{R}}-1\right)\left[\varphi_{0}\left(z_{\mathrm{I}}^{\mathrm{R}}\right)\right]^{2} \\
& \times \Delta E_{\mathrm{c}}^{\mathrm{R}} .
\end{aligned}
$$

First we assume, for simplicity, that the wave function vanishes at the left heterointerface, that is, $\varphi_{0}\left(z_{\mathrm{I}}^{\mathrm{L}}\right)=0$ [the numerically calculated wave function, shown in Fig. 3(b), is reduced considerably at the left heterointerface by applying a large negative $E_{z}^{\mathrm{ex}}$ although it does not vanish completely]. In this case, from Eq. (16), we have $\alpha=\eta\left(b_{\mathrm{off}}^{\mathrm{R}}-1\right)\left\langle 0\left|\left(\nabla_{z} V_{\mathrm{bo}}^{\mathrm{cR}}\right)\right| 0\right\rangle$, which vanishes at $b_{\mathrm{off}}^{\mathrm{R}}=1$. In Fig. 2 , we plot $b_{\mathrm{off}}^{\mathrm{R}}$ for $\mathrm{S}_{\mathrm{W}}=$ $\mathrm{Ga}_{0.47} \mathrm{In}_{0.53} \mathrm{As}$ and $\mathrm{S}_{\mathrm{B}}^{\mathrm{R}}=\mathrm{Al}_{x} \mathrm{Ga}_{1-x} \mathrm{As}_{y} \mathrm{Sb}_{1-y}$ as a function of the Al fraction $x$ in $\mathrm{S}_{\mathrm{B}}^{\mathrm{R}}$ where $y$ is determined so that $\mathrm{S}_{\mathrm{B}}^{\mathrm{R}}$ is lattice-matched to $\mathrm{S}_{\mathrm{W}}$ [16]. This figure shows that $b_{\text {off }}^{\mathrm{R}}=1$ at a value $x_{0}$ which is close to $x=0.3$ for the values of band parameters from Ref. [30]. Therefore, an asymmetric quantum well consisting of $\mathrm{S}_{\mathrm{W}}=\mathrm{Ga}_{0.47} \mathrm{In}_{0.53} \mathrm{As}, \mathrm{S}_{\mathrm{B}}^{\mathrm{L}}=\mathrm{Al}_{0.48} \mathrm{In}_{0.52} \mathrm{As}$ $\left(b_{\text {off }}^{\mathrm{L}}=-0.32\right)$, and $\mathrm{S}_{\mathrm{B}}^{\mathrm{R}}=\mathrm{Al}_{x} \mathrm{Ga}_{1-x} \mathrm{As}_{y} \mathrm{Sb}_{1-y}$ with $x=x_{0}$ $\left(b_{\mathrm{off}}^{\mathrm{R}}=1\right)$ has $|\alpha| \approx 0$ for a large negative $E_{z}^{\mathrm{ex}}$ where $\varphi_{0}\left(z_{\mathrm{I}}^{\mathrm{L}}\right)$ is considerably reduced, while it acquires a substantial value of $|\alpha|$ for a large positive $E_{z}^{\text {ex }}$ where $\varphi_{0}\left(z_{\mathrm{I}}^{\mathrm{L}}\right)$ is increased. This means that $|\alpha|$ is switched on and off by changing the polarity of $E_{z}^{\mathrm{ex}}$.

Now we evaluate the on/off ratio of $|\alpha|$, denoted by $R_{\alpha}$, when $x$ slightly deviates from $x_{0}$. We start from the expression of $\alpha$ in the case of $\varphi_{0}\left(z_{\mathrm{I}}^{\mathrm{L}}\right)=$ (a)

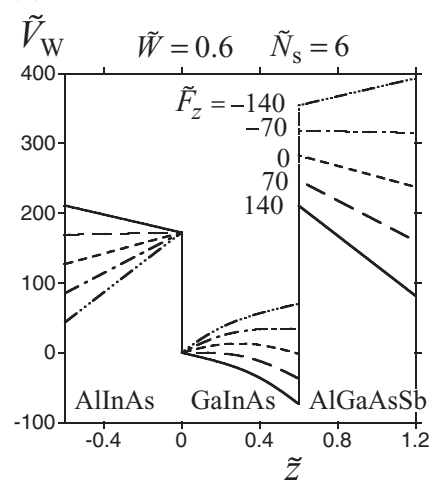

(b)

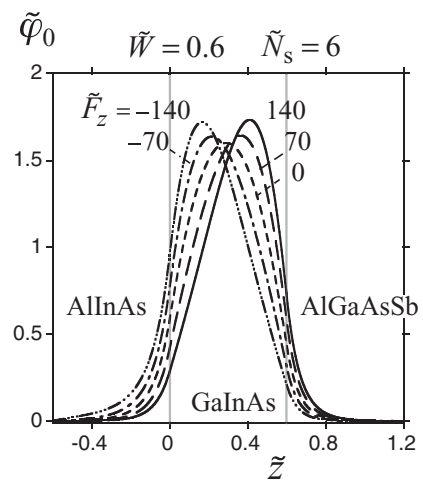

FIG. 3. Calculated results of (a) $V_{\mathrm{W}}(z)$ and (b) $\varphi_{0}(z)$ for different values of the external force $F_{z}=-e E_{z}^{\mathrm{ex}}$ in $\mathrm{Al}_{0.48} \mathrm{In}_{0.52} \mathrm{As} / \mathrm{Ga}_{0.47} \mathrm{In}_{0.53} \mathrm{As} / \mathrm{Al}_{x} \mathrm{Ga}_{1-x} \mathrm{As}_{y} \mathrm{Sb}_{1-y}(x=0.3)$. The parameter $W\left(=z_{\mathrm{I}}^{\mathrm{R}}-z_{\mathrm{I}}^{\mathrm{L}}\right)$ is the well width. The dimensionless quantities are defined by $\tilde{V}_{\mathrm{W}}=V_{\mathrm{W}} / \mathrm{Ry}^{*}, \quad \tilde{\varphi}_{0}=\varphi_{0}\left(a_{B}^{*}\right)^{1 / 2}, \quad \tilde{z}=z / a_{B}^{*}, \quad \tilde{F}_{z}=$ $F_{z} /\left(\mathrm{Ry}^{*} / a_{B}^{*}\right), \tilde{W}=W / a_{B}^{*}$, and $\tilde{N}_{\mathrm{s}}=N_{\mathrm{s}}\left(a_{B}^{*}\right)^{2}$ with $a_{B}^{*}=\hbar^{2} \epsilon /\left(m e^{2}\right)$ and $\mathrm{Ry}^{*}=\hbar^{2}\left(a_{B}^{*}\right)^{-2} /(2 m)$. For $\mathrm{Ga}_{0.47} \mathrm{In}_{0.53} \mathrm{As}, a_{B}^{*}=17.1 \mathrm{~nm}$ and $\mathrm{Ry}^{*}=3.04 \mathrm{meV}$. Therefore $\tilde{W}=0.6$ and $\tilde{N}_{\mathrm{s}}=6$ correspond to $W=10.3 \mathrm{~nm}$ and $N_{\mathrm{s}}=2.05 \times 10^{16} \mathrm{~m}^{-2}$.

$0, \quad \alpha=\eta\left(b_{\mathrm{off}}^{\mathrm{R}}-1\right)\left\langle 0\left|\left(\nabla_{z} V_{\mathrm{bo}}^{\mathrm{cR}}\right)\right| 0\right\rangle . \quad$ By using $\varphi_{0}\left(z_{\mathrm{I}}^{\mathrm{L}}\right)=0$ as well as $\left\langle 0\left|\left(\nabla_{z} V_{\mathrm{W}}\right)\right| 0\right\rangle=0$ and $\left\langle 0\left|\left(\nabla_{z} V_{\mathrm{es}}\right)\right| 0\right\rangle=e E_{z}^{\mathrm{ex}}$, we have $\left\langle 0\left|\left(\nabla_{z} V_{\mathrm{bo}}^{\mathrm{cR}}\right)\right| 0\right\rangle=\left\langle 0\left|\left(\nabla_{z} V_{\mathrm{bo}}^{\mathrm{c}}\right)\right| 0\right\rangle=\left\langle 0\left|\left(-\nabla_{z} V_{\mathrm{es}}\right)\right| 0\right\rangle=$ $-e E_{z}^{\mathrm{ex}}$ and then obtain $\alpha=\eta\left(b_{\mathrm{off}}^{\mathrm{R}}-1\right)\left(-e E_{z}^{\mathrm{ex}}\right)$. Similarly we obtain $\alpha=\eta\left(b_{\mathrm{off}}^{\mathrm{L}}-1\right)\left(-e E_{z}^{\mathrm{ex}}\right)$ in the case of $\varphi_{0}\left(z_{\mathrm{I}}^{\mathrm{R}}\right)=0$. Then the on/off ratio $R_{\alpha}$, when the polarity of $E_{z}^{\mathrm{ex}}$ is changed with a large enough $\left|E_{z}^{\mathrm{ex}}\right|$, becomes

$$
R_{\alpha} \equiv \frac{|\alpha|_{\text {on }}}{|\alpha|_{\text {off }}}=\frac{\left|b_{\text {off }}^{\mathrm{L}}-1\right|}{\left|b_{\text {off }}^{\mathrm{R}}-1\right|} .
$$

For a deviation of $\left|x-x_{0}\right|=0.01$, we have $\left|b_{\text {off }}^{\mathrm{R}}-1\right| \approx 1 / 30$ from Fig. 2. Therefore we obtain $R_{\alpha} \approx 40$, which leads to the on/off ratio of the spin-relaxation rate $\left(R_{\mathrm{sr}}\right)$ exceeding $10^{3}$ in the Dyakonov-Perel mechanism where $R_{\mathrm{sr}}=\left(R_{\alpha}\right)^{2}$.

In order to examine how large $\left|E_{z}^{\mathrm{ex}}\right|$ is necessary to realize the on/off $|\alpha|$ ratio $R_{\alpha}$ in $\mathrm{Eq}$. (17), we perform a numerical calculation for $\varphi_{0}(z)$ by solving the Schrödinger equation Eq. (1) and the Poisson equation Eq. (3) self-consistently, in which we discretize the $z$ coordinate. The obtained $\varphi_{0}(z)$ as well as $V_{\mathrm{W}}(z)$ in $\mathrm{Al}_{0.48} \mathrm{In}_{0.52} \mathrm{As} / \mathrm{Ga}_{0.47} \mathrm{In}_{0.53} \mathrm{As} / \mathrm{Al}_{x} \mathrm{Ga}_{1-x} \mathrm{As}_{y} \mathrm{Sb}_{1-y} \quad(x=0.3)$ are plotted in Fig. 3 for five different values of the external force $F_{z}=-e E_{z}^{\mathrm{ex}}$ (the energy separation between the ground subband and the first-excited subband increases with $\left|E_{z}^{\mathrm{ex}}\right|$ and the excited subbands are not occupied by electrons in the cases we considered in this paper), while the value of $\alpha$ as a function of $F_{z}$ is presented in Fig. 4 for three different quantum wells. All of the three quantum wells have a common material in the well layer, $\mathrm{Ga}_{0.47} \operatorname{In}_{0.53}$ As. The difference of the three is in the combination of interfaces, LL, SS, and LS, where L $\{\mathrm{S}\}$ is the interface which gives a large $\{$ small $\}\left|b_{\text {off }}-1\right|$, $\mathrm{Ga}_{0.47} \mathrm{In}_{0.53} \mathrm{As}$ (well)/Al $\mathrm{Al}_{0.48} \mathrm{In}_{0.52} \mathrm{As}$ (barrier) with $b_{\text {off }}-1=$ $-1.32\left\{\mathrm{Ga}_{0.47} \mathrm{In}_{0.53} \mathrm{As}\right.$ (well)/Al $\mathrm{Al}_{x} \mathrm{Ga}_{1-x} \mathrm{As}_{y} \mathrm{Sb}_{1-y}$ (barrier) $(x=0.3)$ with $\left.b_{\text {off }}-1=-0.05\right\}$. 

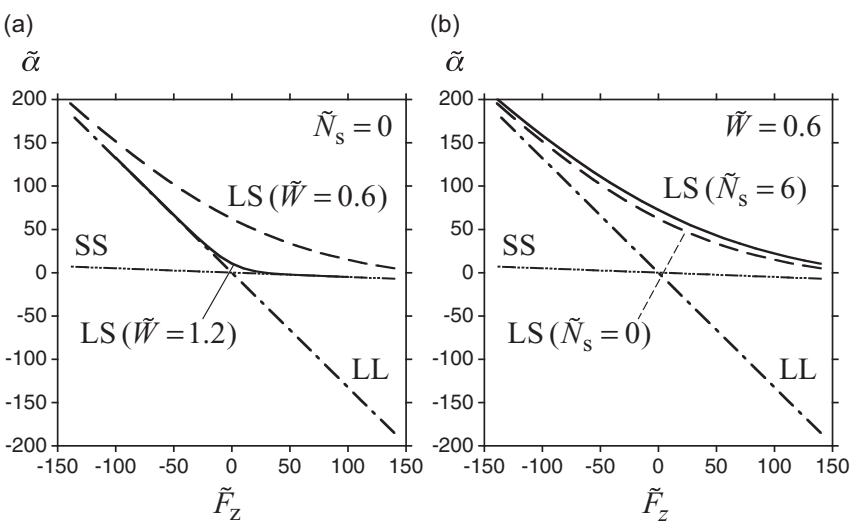

FIG. 4. Rashba coefficient $\alpha$ as a function of the external force $F_{z}=-e E_{z}^{\mathrm{ex}}$ for three combinations of interfaces: LL, SS, and LS (see text). (a) The well-width ( $W$ ) dependence for LS. (b) The electronsheet-density $\left(N_{\mathrm{S}}\right)$ dependence for LS. The dimensionless Rashba coefficient is defined by $\tilde{\alpha}=(\alpha / \eta) /\left(\mathrm{Ry}^{*} / a_{B}^{*}\right)$. For the definition of other quantities, see the caption of Fig. 3.

In the case of $b_{\text {off }}^{\mathrm{L}}=b_{\text {off }}^{\mathrm{R}}\left(=b_{\text {off }}\right)$ as in LL and SS, Eq. (16) becomes $\alpha=\eta\left(b_{\text {off }}-1\right)\left\langle 0\left|\left(\nabla_{z} V_{\text {bo }}^{\mathrm{c}}\right)\right| 0\right\rangle=-\eta\left(b_{\text {off }}-\right.$ 1) $\left\langle 0\left|\left(\nabla_{z} V_{\mathrm{es}}\right)\right| 0\right\rangle=\eta\left(b_{\mathrm{off}}-1\right) F_{z}$, where we have used the equalities $\left\langle 0\left|\left(\nabla_{z} V_{\mathrm{W}}\right)\right| 0\right\rangle=0$ and $\left\langle 0\left|\left(\nabla_{z} V_{\mathrm{es}}\right)\right| 0\right\rangle=e E_{z}^{\mathrm{ex}}=$ $-F_{z}$. Therefore $\alpha$ of LL and that of SS are independent of the well width $W$ and $N_{\mathrm{s}}$. They are plotted in Fig. 4 as straight lines with different gradients.

The calculated $\alpha\left(F_{z}\right)$ of LS, presented in Fig. 4, approaches the steep-slope $\alpha\left(F_{z}\right)$ of LL in $F_{z}<0$ and the gentle-slope $\alpha\left(F_{z}\right)$ of SS in $F_{z}>0$ as $\left|F_{z}\right|$ increases, which demonstrates the switching of $|\alpha|$ by changing the sign of $F_{z}$ ( $\alpha$ of LS deviates from zero at $F_{z}=0$, which is derived in [31]). A rough estimate for the value of $\left|F_{z}\right|$, at which $\alpha\left(F_{z}\right)$ of LS merges with that of LL or SS, denoted by $F_{z}^{c}$, is given by the potential difference between two interfaces $e\left|E_{z}^{\text {ex }}\right| W=$ $\left|F_{z}\right| W$ equal to the energy difference between the ground subband and the first excited subband at $N_{\mathrm{s}}=0$ and $E_{z}^{\mathrm{ex}}=0$ in the infinite-barrier model $\varepsilon_{1}-\varepsilon_{0}=3 \hbar^{2} \pi^{2} /\left(2 m W^{2}\right)$. This estimate shows a strong $W$ dependence of $F_{z}^{c}: F_{z}^{c} \propto W^{-3}$, which explains the $W$ dependence (at $N_{\mathrm{s}}=0$ ) presented in Fig. 4(a). Using dimensionless variables, $\tilde{F}_{z}^{c}=F_{z}^{c} /\left(\mathrm{Ry}^{*} / a_{B}^{*}\right)$ and $\quad \tilde{W}=W / a_{B}^{*} \quad$ with $\quad a_{B}^{*}=\hbar^{2} \epsilon /\left(m e^{2}\right) \quad$ and $\quad \mathrm{Ry}^{*}=$ $\hbar^{2}\left(a_{B}^{*}\right)^{-2} /(2 m)$, such an estimate is expressed as $\tilde{F}_{z}^{c}=3 \pi^{2} \tilde{W}^{-3}$, which gives $\tilde{F}_{z}^{c}=137$ for $\tilde{W}=0.6$ and $\tilde{F}_{z}^{c}=17.1$ for $\tilde{W}=1.2$. This estimate for $\tilde{F}_{z}^{c}$ is consistent with that extracted from Fig. 4(a). The corresponding value of $\left|E_{z}^{\mathrm{ex}}\right|$, denoted by $E_{z}^{c}$, becomes $E_{z}^{c}=2.4 \times 10^{7} \mathrm{~V} / \mathrm{m}$ $(W=10.3 \mathrm{~nm})$ and $E_{z}^{c}=3.0 \times 10^{6} \mathrm{~V} / \mathrm{m}(W=20.5 \mathrm{~nm})$ when $a_{B}^{*}=17.1 \mathrm{~nm}$ and $\mathrm{Ry}^{*}=3.04 \mathrm{meV}$ for $\mathrm{Ga}_{0.47} \mathrm{In}_{0.53} \mathrm{As}$ is used. On the other hand, the dependence of $\alpha$ on the electron sheet density $N_{\mathrm{s}}$ is weak as shown in Fig. 4(b). We therefore find that $\left|E_{z}^{\mathrm{ex}}\right|$ above $E_{z}^{c}=3 \pi^{2} \tilde{W}^{-3} \mathrm{Ry}^{*} /\left(e a_{B}^{*}\right)$ is required to realize the on/off $|\alpha|$ ratio $R_{\alpha}$ given by Eq. (17). From the numerical result in Fig. 4(a) showing that $\alpha$ of LS for $\tilde{W}=1.2$ is well approximated by $\alpha$ of $\operatorname{LL}\left(F_{z}<0\right)$ and $\alpha$ of SS $\left(F_{z}>0\right)$ at $\left|\tilde{F}_{z}\right|>50$, it is derived that $R_{\alpha}$ reaches the value in Eq. (17) at $\left|E_{z}^{\text {ex }}\right|>10^{7} \mathrm{~V} / \mathrm{m}$ for $W=20.5 \mathrm{~nm}$ $\left(\left|E_{z}^{\mathrm{ex}}\right|=3 \times 10^{7} \mathrm{~V} / \mathrm{m}\right.$ has been experimentally attained [32]).

In conclusion, we have calculated the coefficient of the Rashba spin-orbit interaction, $\alpha$, as a function of the external electric field perpendicular to the well, $E_{z}^{\mathrm{ex}}$, in an asymmetric quantum well consisting of $\mathrm{Ga}_{0.47} \mathrm{In}_{0.53} \mathrm{~A} s$ (well), $\mathrm{Al}_{0.48} \mathrm{In}_{0.52}$ As (left barrier), and $\mathrm{Al}_{0.3} \mathrm{Ga}_{0.7} \mathrm{As}_{y} \mathrm{Sb}_{1-y}$ (right barrier). We have found that $|\alpha|$ and the resulting spinrelaxation rate in the Dyakonov-Perel mechanism can be switched on and off by changing the polarity of $E_{z}^{\mathrm{ex}}$ when $\left|E_{z}^{\mathrm{ex}}\right|$ is large enough. The required $\left|E_{z}^{\text {ex }}\right|$ is proportional to $W^{-3}$ with $W$ the well width, and weakly depends on the electron sheet density.

The Dresselhaus spin-orbit interaction [33] also contributes to the spin relaxation in the Dyakonov-Perel mechanism in addition to the Rashba spin-orbit interaction which has been considered in this paper. However, in a quantum well parallel to the (110) plane of the zinc-blende structure, the effective magnetic field induced by the Dresselhaus spin-orbit interaction is perpendicular to the well layer and does not give the spin relaxation in the Dyakonov-Perel mechanism for the spin component perpendicular to the well layer. This has been shown theoretically in [15] and the resulting reduction in the spin relaxation rate has been demonstrated experimentally in [34-37]. Therefore the estimate of the spin relaxation rate, given in this paper, does not change in (110) quantum wells for the perpendicular spin component even when the Dresselhaus spin-orbit interaction is considered in addition to the Rashba spin-orbit interaction.
[1] I. Žutić, J. Fabian, and S. D. Sarma, Rev. Mod. Phys. 76, 323 (2004).

[2] D. D. Awschalom and M. E. Flatté, Nat. Phys. 3, 153 (2007).

[3] S. Datta and B. Das, Appl. Phys. Lett. 56, 665 (1990).

[4] E. I. Rashba, Sov. Phys. Solid State 2, 1109 (1960).

[5] F. J. Ohkawa and Y. Uemura, J. Phys. Soc. Jpn. 37, 1325 (1974).

[6] Y. A. Bychkov and E. I. Rashba, J. Phys. C 17, 6039 (1984).

[7] Y. A. Bychkov and E. I. Rashba, JETP Lett. 39, 78 (1984).

[8] J. Nitta, T. Akazaki, H. Takayanagi, and T. Enoki, Phys. Rev. Lett. 78, 1335 (1997).

[9] G. Engels, J. Lange, T. Schäpers, and H. Lüth, Phys. Rev. B 55, R1958 (1997).
[10] T. Koga, J. Nitta, T. Akazaki, and H. Takayanagi, Phys. Rev. Lett. 89, 046801 (2002).

[11] K. C. Hall, W. H. Lau, K. Gündoğdu, M. E. Flatté, and T. F. Boggess, Appl. Phys. Lett. 83, 2937 (2003).

[12] K. C. Hall and M. E. Flatté, Appl. Phys. Lett. 88, 162503 (2006).

[13] M. I. Dyakonov and V. I. Perel, Sov. Phys. JETP 33, 1053 (1971).

[14] M. I. Dyakonov and V. I. Perel, Sov. Phys. Solid State 13, 3023 (1972).

[15] M. I. Dyakonov and V. Y. Kachorovskii, Sov. Phys. Semicond. 20, 110 (1986).

[16] H. Akera, H. Suzuura, and Y. Egami, Phys. Rev. B 92, 205311 (2015). 
[17] E. A. de Andrada e Silva, G. C. La Rocca, and F. Bassani, Phys. Rev. B 55, 16293 (1997). This paper describes a theory on the spin-orbit splitting for an asymmetric quantum well $\mathrm{Al}_{x} \mathrm{Ga}_{1-x} \mathrm{As} / \mathrm{GaAs} / \mathrm{Al}_{y} \mathrm{Ga}_{1-y} \mathrm{As}(x \neq y)$ in the absence of the electrostatic potential $\left[V_{\mathrm{es}}(z)=0\right]$.

[18] R. Lassnig, Phys. Rev. B 31, 8076 (1985).

[19] R. S. Calsaverini, E. Bernardes, J. C. Egues, and D. Loss, Phys. Rev. B 78, 155313 (2008).

[20] T. Schäpers, G. Engels, J. Lange, T. Klocke, M. Hollfelder, and H. Lüth, J. Appl. Phys. 83, 4324 (1998).

[21] L. Wissinger, U. Rössler, R. Winkler, B. Jusserand, and D. Richards, Phys. Rev. B 58, 15375 (1998).

[22] P. Pfeffer, Phys. Rev. B 59, 15902 (1999).

[23] P. Pfeffer and W. Zawadzki, Phys. Rev. B 68, 035315 (2003).

[24] M. O. Nestoklon, S. A. Tarasenko, J.-M. Jancu, and P. Voisin, Phys. Rev. B 85, 205307 (2012).

[25] E. O. Kane, J. Phys. Chem. Solids 1, 249 (1957).

[26] A. Därr, J. P. Kotthaus, and T. Ando, in Proceedings of the 13th International Conference on the Physics of Semiconductors, Rome, edited by F. G. Fumi (North-Holland, Amsterdam, 1976), p. 774.

[27] T. Ando, A. B. Fowler, and F. Stern, Rev. Mod. Phys. 54, 437 (1982).

[28] W. Zawadzki and P. Pfeffer, Phys. Rev. B 64, 235313 (2001).
[29] From Eq. (1) $\varphi_{0}(z)=\langle z \mid 0\rangle$ is the eigenfunction corresponding to the eigenvalue $\varepsilon_{0}$ of the Hamiltonian $\hat{H}=\hat{p}_{z}^{2} /(2 m)+$ $V_{\mathrm{W}}(z)$ with $\left[\hat{p}_{z}, \hat{H}\right]=-i \hbar\left(\nabla_{z} V_{\mathrm{W}}\right)$. Since $\left\langle 0\left|\left[\hat{p}_{z}, \hat{H}\right]\right| 0\right\rangle=$ $\left\langle 0\left|\hat{p}_{z}\right| 0\right\rangle\left(\varepsilon_{0}-\varepsilon_{0}\right)=0$, we obtain $\left\langle 0\left|\left(\nabla_{z} V_{\mathrm{W}}\right)\right| 0\right\rangle=0$.

[30] I. Vurgaftman, J. R. Meyer, and L. R. Ram-Mohan, J. Appl. Phys. 89, 5815 (2001).

[31] $\alpha$ in Eq. (16) can also be written as the sum of a term proportional to $F_{z}\left(=-e E_{z}^{\mathrm{ex}}\right)$ and that proportional to $b_{\mathrm{off}}^{\mathrm{L}}-b_{\mathrm{off}}^{\mathrm{R}}$ :

$$
\alpha=\eta\left(b_{\mathrm{off}}^{\mathrm{av}}-1\right) F_{z}+\eta\left(b_{\mathrm{off}}^{\mathrm{L}}-b_{\mathrm{off}}^{\mathrm{R}}\right)\left\langle 0\left|\left(\nabla_{z} \frac{V_{\mathrm{bo}}^{\mathrm{cL}}-V_{\mathrm{bo}}^{\mathrm{cR}}}{2}\right)\right| 0\right\rangle,
$$

with $b_{\text {off }}^{\text {av }}=\left(b_{\text {off }}^{\mathrm{L}}+b_{\text {off }}^{\mathrm{R}}\right) / 2$. At $F_{z}=0$ the first term is zero, while the second term is nonzero in an asymmetric quantum well with $b_{\text {off }}^{\mathrm{L}} \neq b_{\text {off }}^{\mathrm{R}}$.

[32] S. Faniel, T. Matsuura, S. Mineshige, Y. Sekine, and T. Koga, Phys. Rev. B 83, 115309 (2011).

[33] G. Dresselhaus, Phys. Rev. 100, 580 (1955).

[34] Y. Ohno, R. Terauchi, T. Adachi, F. Matsukura, and H. Ohno, Phys. Rev. Lett. 83, 4196 (1999).

[35] T. Adachi, Y. Ohno, F. Matsukura, and H. Ohno, Physica E 10, 36 (2001).

[36] K. C. Hall, K. Gündoğdu, E. Altunkaya, W. H. Lau, M. E. Flatté, T. F. Boggess, J. J. Zinck, W. B. Barvosa-Carter, and S. L. Skeith, Phys. Rev. B 68, 115311 (2003).

[37] G. M. Müller, M. Römer, D. Schuh, W. Wegscheider, J. Hübner, and M. Oestreich, Phys. Rev. Lett. 101, 206601 (2008). 